\title{
Segmentation of hand radiographs by using multi-level connected active appearance models
}

\author{
J.A. Kauffman ${ }^{1}$, C.H. Slump ${ }^{1}$, H.J. Bernelot Moens ${ }^{2}$
}

Signals and Systems Group

Department of Electrical Engineering, Mathematics and Computer Science

University of Twente, Enschede, http://www.sas.el.utwente.nl, email: j.a.kauffman@utwente.nl

Department of Rheumatology

Ziekenhuis Groep Twente, Hengelo/Almelo

University of Twente

The Netherlands

\section{Introduction}

Rheumatoid arthritis

- chronic inflammatory disease causing joint damage

- hand radiographs used for monitoring disease progression

Goal: locate the bones in a hand radiograph

Problems

- variabilities in finger positioning

- artifacts: rings, labels, implants, etc.

- present joint damage

Methods

- active appearance

model (AAM) [1]

- connected AAMs [2]

- iterative AAM search

- multiple search levels

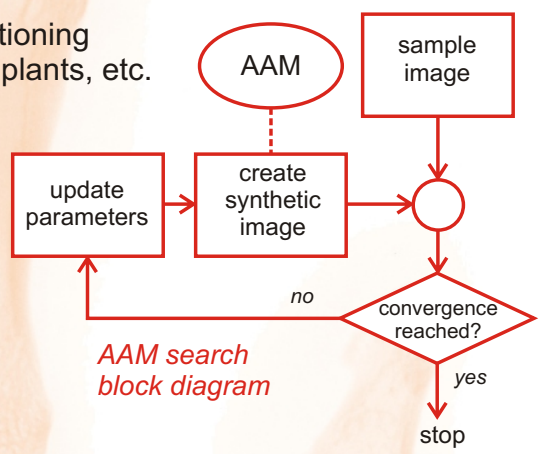

\section{Modeling the hand}

Compensate for variability caused by

differences in hand positioning

- use sub-AAM for each bone

- define structure of subcoordinate

frames

- within each frame: make submodels

invariant to rotation and translation

- connect sub-AAMs in a single

parametric model

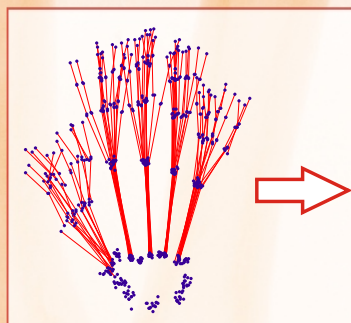

positioning variabilities in 15 sample images

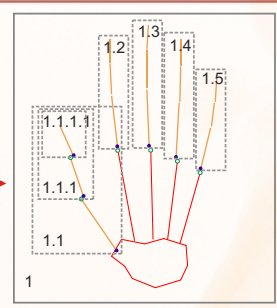

subcoordinate frames

positioning variabilities removed

\section{AAM search}

Multiple levels

- begin with a low detail model: few landmarks and low texture resolution

- initialize at center of gravity

- after search convergence, use parameters to initialize the next search with a more detailed model

\section{Training set}

Connected AAMs:

- 50 digitized film radiographs

- manually outlined contours

- 'fish bone' shaped grid for defining 64 landmarks (LMs) on metacarpals, proximal and middle phalanges

- 10 LMs for carpal region and 4 LMs for distal phalanges
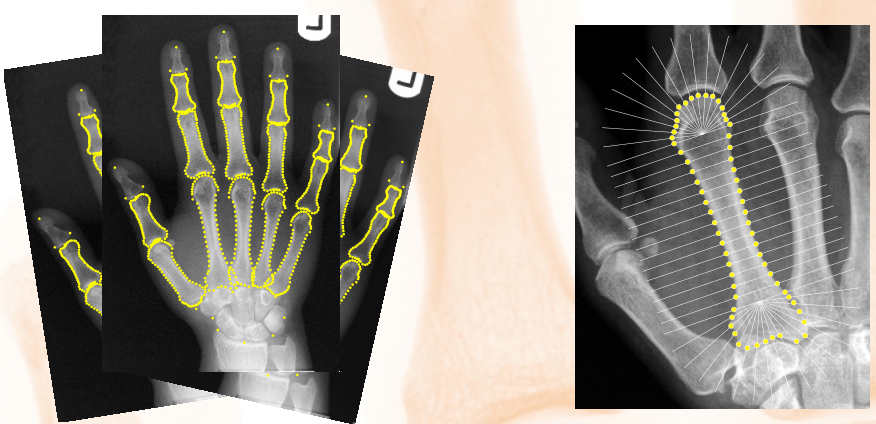

\section{Results}

Test setup

- 30 test images

- two search levels,

first: 4 LMs per submodel at 8 pixels $/ \mathrm{cm}$

second: up to $64 \mathrm{LMs}$ per submodel at 15 pixels/cm

- detected landmark locations are compared to manually placed landmarks

Results

- mean error $<1.3 \mathrm{~mm}$ for 22 images

- large failures in 2 images caused by poor initialization

- mean error < $4.3 \mathrm{~mm}$ for remaining 6 images

Performance

- fewer errors and faster compared to a single level search

- smaller errors than without rotation and translation invariant submodels

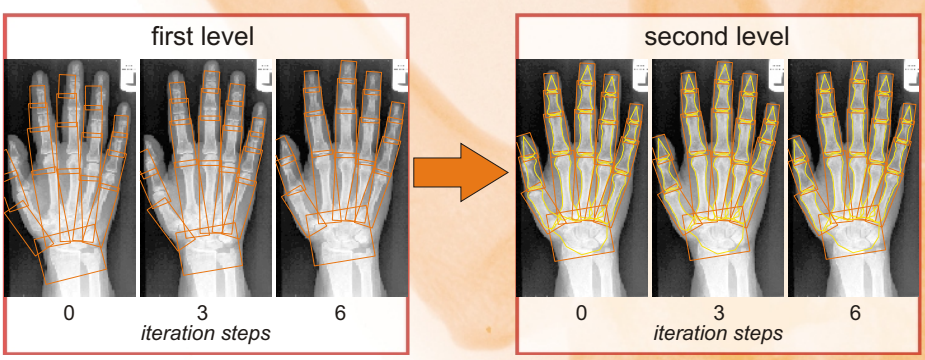

\section{Conclusions}

- The use of multiple search levels reduces search time and improves segmentation results.

- Making the model invariant to lateral finger displacements results in smaller segmentation errors.

- Improvement of the initialization of the AAM search may lead to fewer search errors.

\section{References}

[1] Cootes, T. F., Edwards, G. J., and Taylor, C. J., "Active appearance models," IEEE Transactions on Pattern Analysis and Machine Intelligence, vol. 23, no. 6, pp. 681-685, 2001.

[2] Bukovec, M., Truyen, R., Likar, B., Bernard, R., and Pernus, F. Image Segmentation by Connected Parametrical Models. Medical Imaging 2004: Image Processing 5370, 398-409. 2004. SPIE. 